\title{
Bifunktionaler Sensorchip für den Nachweis von Gelöstwasserstoff
}

\author{
Christina Huck ${ }^{1,2}$, Matthias Bäcker ${ }^{1,2}$, Arshak Poghossian ${ }^{1,2}$, Patrick Wagner $^{3}$ und Michael J. \\ Schöning ${ }^{1,2}$ \\ ${ }^{1}$ Institut für Nano- und Biotechnologien (INB), FH Aachen, Campus Jülich, Heinrich-Mußmann- \\ Straße 1, D-52428 Jülich \\ ${ }^{2}$ Peter-Grünberg Institut (PGI-8), Forschungszentrum Jülich GmbH, Wilhelm-Johnen-Straße, D- \\ 52525 Jülich \\ ${ }^{3}$ Instituut voor Materiaalonderzoek (IMO), Universiteit Hasselt, Wetenschapspark 1, B-3590 \\ Diepenbeek
}

\section{Zusammenfassung}

In diesem Beitrag wird ein neuartiger bifunktionaler Sensorchip für den Nachweis von Gelöstwasserstoff vorgestellt. Die Besonderheit des Sensorchips zeichnet sich durch die Kombination aus einem feldeffektbasierten $\mathrm{pH}-$ Sensor für den indirekten Nachweis von Gelöstwasserstoff mit einem amperometrischen Sensor aus. Die Realisierung dieser beiden Sensorprinzipien vereint auf einem Chip wird in der vorliegenden Arbeit anhand einer amperometrischen Messung von Gelöstwasserstoff unter gleichzeitiger Aufnahme von Kapazitäts-/Spannungs-Kurven (C/V-Kurven) und deren Verschiebung in Abhängigkeit der Wasserstoffkonzentration demonstriert.

\section{Einleitung}

Die Biogasgewinnung etabliert sich zunehmend in der Gruppe der alternativen Energien, wenn es darum geht, verlässlich, nachhaltig und unabhängig von fossilen Energieträgern oder den oft in der Kritik stehenden nuklearen Anlagen, Energie zu produzieren. Die Einhaltung optimaler Prozessparameter ist für die Biogasproduktion von besonderer Bedeutung. Bei nicht idealen Milieubedingungen wird nicht nur die Produktivität der Biogaserzeugung gesenkt, ungünstige Milieubedingungen können im schlimmsten Fall den Absturz des Gesamtprozesses auslösen. Tritt dieser Fall ein, muss der Inhalt der Anlage entsorgt und die Anlage gereinigt werden. Damit verbunden sind teilweise langwierige Produktionsausfälle, welche die eigentlichen Vorteile der Energiegewinnung mittels Biogas konterkarieren [1].

Vor diesem Hintergrund ist der Einsatz zeitnaher Kontrollmethoden, die einen frühzeitigen Eingriff in den Fermentationsprozess gestatten, von hohem ökonomischem und ökologischem Interesse. Einer der wichtigsten Parameter ist die Gelöstwasserstoffkonzentration des Biogasmediums, da diese eine Schlüsselrolle in dem komplexen synergetischen Zusammenspiel einnimmt und die aktuelle Belastung des Reaktors mit hoher Sensitivität beschreibt. Wasserstoff ist ein Zwischenprodukt der anaeroben Vergärung, das den Abbau von Propionsäure energetisch hemmt, sobald der Partialdruck einen Grenzwert von ca. 6 Pa überschreitet. Ein Anstieg der Wasserstoffkonzentration deutet somit auf ein Ungleichgewicht der Abbauschritte hin, das eine Akkumulation von Propionsäure und damit eine Prozesshemmung zur Folge hat [2].

Im Rahmen der vorliegenden Arbeit wird erstmals ein Sensorsystem zur Gelöstwasserstoffmessung vorgestellt, welches sowohl auf dem amperometrischen Prinzip als auf der Feldeffektmessung beruht. Die Kombination dieser beiden Transducerprinzipien auf einem Chip für gleichzeitige Parameterbestimmung könnte in Zukunft eine selektivere und zuverlässigere Messung von Gelöstwasserstoff als Frühwarnindikator für die Biogasüberwachung ermöglichen. 


\section{Experimentelles}

\subsection{Sensorherstellung}

In Bild 1 ist die schematische Struktur des bifunktionalen chipbasierten Gelöstwasserstoffsensors dargestellt.

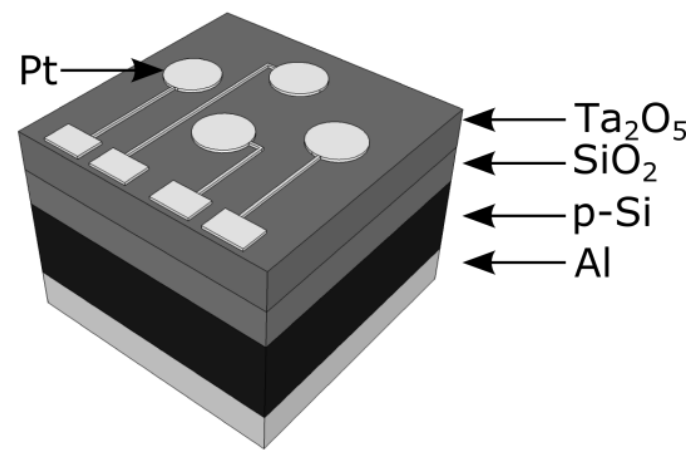

Bild 1 Schematische Darstellung des bifunktionalen chipbasierten Gelöstwasserstoffsensors.

Der kapazitive Elektrolyt-Isolator-Halbleiter (EIS)-Sensor besteht aus einer Al-Si-SiO${ }_{2}-\mathrm{Ta}_{2} \mathrm{O}_{5}$-Struktur. Für die Herstellung wurde auf die Oberfläche von p-Si (1-10 $\Omega \mathrm{cm}) 30 \mathrm{~nm} \mathrm{SiO}{ }_{2}$ mittels thermischer Oxidation gewachsen. Anschließend wurden $30 \mathrm{~nm}$ Tantal durch Elektronenstrahlverdampfung abgeschieden und in Sauerstoffatmosphäre bei $517^{\circ} \mathrm{C}$ für $30 \mathrm{~min}$ oxidiert. Daraus resultiert eine $60 \mathrm{~nm}$ dicke $\mathrm{Ta}_{2} \mathrm{O}_{5}$-Schicht. $\mathrm{Ta}_{2} \mathrm{O}_{5}$ als pH-sensitiver Gate-Isolator ist allgemein bekannt für seine hervorragenden Eigenschaften in Bezug auf feldeffektbasierte $\mathrm{pH}-$ Messungen. Es hat sowohl eine nahezu Nernst ${ }^{\star}$ sche $\mathrm{pH}$-Sensitivität als auch eine hohe Korrosionsunempfindlichkeit in einem weitem pH-Bereich [3,4]. Abschließend wurde $300 \mathrm{~nm}$ Aluminium als Rückenseitenkontakt für den feldeffektbasierten Sensorteil abgeschieden.

Für die amperometrischen Messungen wurden $20 \mathrm{~nm}$ Titan als Haftvermittler und $200 \mathrm{~nm}$ Platin als Elektrodenmaterial mittels Elektronenstrahlverdampfung abgeschieden und durch Photolithographie und Lift-off Prozess strukturiert. Zum Schluss wurde der Wafer zu Chips mit einer Chipgröße von $10 \mathrm{~mm}$ x $10 \mathrm{~mm}$ vereinzelt.

Zur elektrischen Kontaktierung wurde der Al-Rückseitenkontakt des EIS-Sensors durch elektrisch leitenden Kleber in ein PCB-Substrat (printed circuit board) eingebracht. Der Vorderseitenkontakt der vier PlatinDünnschichtelektroden erfolgte durch Ultraschallbonden. Abschließend wurden die Bondflächen mit Silikon verkapselt.

\subsection{Messaufbau und Untersuchungsmethodik}

Für die Untersuchungen wurde das PCB-Substrat mit dem bifunktionalen Sensorchip in eine selbstkonstruierte Messzelle eingeschraubt und über einen O-Ring abgedichtet. Der O-Ring begrenzt somit die Sensorfläche des kapazitiven EIS-Sensorteils. Die Messzelle verfügt sowohl über einen Gaseinlass und auslass als auch einen Zugang für eine konventionelle $\mathrm{Ag} / \mathrm{AgCl}$-Referenzelektrode. WasserstoffStickstoffgemische wurden über kommerzielle Massenflussregler (Bronkhorst High-Tech, Deutschland) in verschiedenen Verhältnissen direkt in den Elektrolyten (0,25 mM Polymix Puffer) eingeleitet und dort gelöst. Die Datenerfassung erfolgte über ein LabVIEW-Programm.

Der feldeffektbasierte pH-Sensor wurde durch ein Impedanzmessgerät (Zahner Elektrik, Deutschland) ausgelesen. Für die Aufnahme der C/V-Kurven wurde eine Gleichspannung zwischen dem AlRückseitenkontakt und der Ag/AgCl-Referenzelektrode angelegt, der eine kleine Wechselspannung ( $20 \mathrm{mV}$, $70 \mathrm{~Hz}$ ) überlagert wurde.

Die elektrochemische Charakterisierung des amperometrischen Gelöstwasserstoffsensors erfolgte in einer Zweielektrodenanordnung unter Verwendung eines Potentiostaten (PalmSens, Niederlande). An die Arbeitselektrode (vier zusammengeschaltete Platinlöffelelektroden) wurde eine Polarisationsspannung von $+0.45 \mathrm{~V}$ versus der $\mathrm{Ag} / \mathrm{AgCl}-$ Referenzelektrode beaufschlagt. 


\section{Ergebnisse und Diskussion}

Für die elektrochemische Charakterisierung des bifunktionalen Gelöstwasserstoffsensors wurden Wasserstoffkonzentrationen im Konzentrationsbereich von 1 bis 3 Vol.-\% dosiert. Bei der amperometrischen Umsetzung von Wasserstoff werden aus jedem Wasserstoffmolekül zwei Elektronen und zwei Protonen generiert $\left(\mathrm{H}_{2} \rightarrow 2 \mathrm{e}^{-}+2 \mathrm{H}^{+}\right)$. Die Elektronen resultieren in einem messbaren Strom in Abhängigkeit der Wasserstoffkonzentration. Dieser proportionale Zusammenhang ist in Bild 2 für den amperometrischen Sensorteil aufgezeigt.

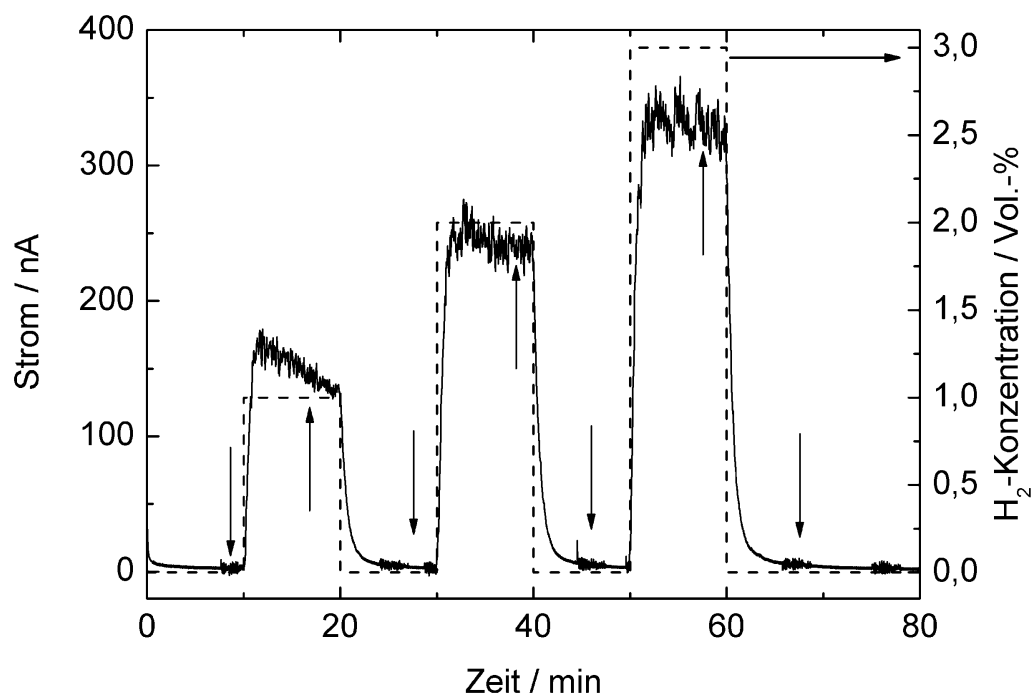

Bild 2 Wasserstoffmessung mit dem amperometrischen Sensorteil des bifunktionalen Gelöstwasserstoffsensors. Die Pfeile kennzeichnen jeweils den Aufnahmezeitpunkt der Kapazitäts-/Spannungs-Kurven.

Die Protonen hingegen führen zu einer lokalen pH-Wertänderung an der Chipoberfläche. Diese sollte vom pH-sensitiven EIS-Sensorteil anhand einer Verschiebung der Kapazitäts-/Spannungs-Kurven (C/V-Kurven) erfasst werden können. Um dies zu untersuchen, wurden gleichzeitig zu der amperometrischen Messung CVKurven unter Stickstoffbegasung und bei verschiedenen Wasserstoffkonzentrationen aufgenommen. Die jeweiligen Aufnahmezeitpunkte der C/V-Kurven sind in Bild 2 anhand von Pfeilen kenntlich gemacht.

In Bild 3 sind die gemessenen C/V-Kurven (links) bei verschiedenen Wasserstoffkonzentrationen dargestellt. Der rechte Teil des Bildes zeigt eine Vergrößerung des Verarmungsbereiches der C/V-Kurven, in der eine
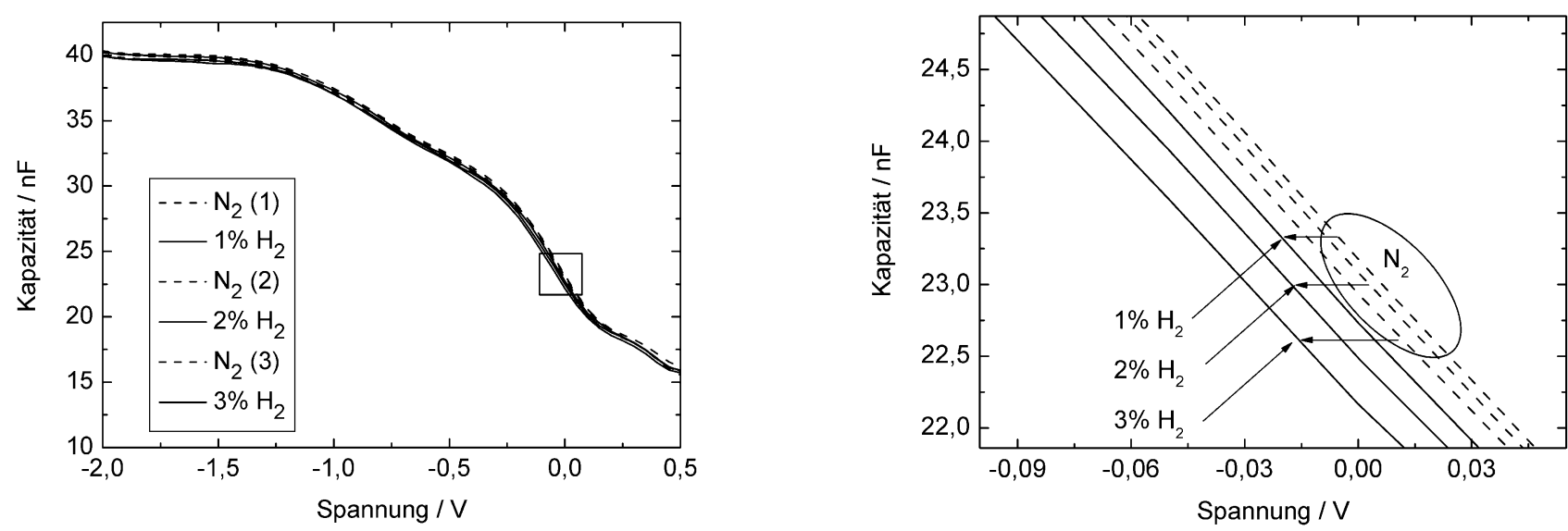

Bild 3 Kapazitäts-/Spannungs-Kurven bei verschiedenen Wasserstoffkonzentrationen (links), Vergrößerung des Verarmungsbereichs (rechts). 
Verschiebung entlang der Spannungsachse zu erkennen ist. Eine zunehmende Wasserstoffkonzentration resultierte in einer Verschiebung der $\mathrm{C} / \mathrm{V}$-Kurve in negative Richtung. Die an der Arbeitselektrode generierten Protonen führen demnach zu einer Ansäuerung, die vom feldeffektbasierten Sensorteil zusätzlich erfasst werden kann.

In Bild 4 ist sowohl die Kalibrierkurve des amperometrischen als auch des feldeffektbasierten Sensorteils gezeigt. Beide Sensorteile wiesen ein lineares Kennlinienverhalten mit einer Sensitivität von $120 \pm 0,2 \mathrm{nA} /$ Vol.-\% bzw. $-6,1 \pm 0,5 \mathrm{mV} /$ Vol.- $\%$ auf.

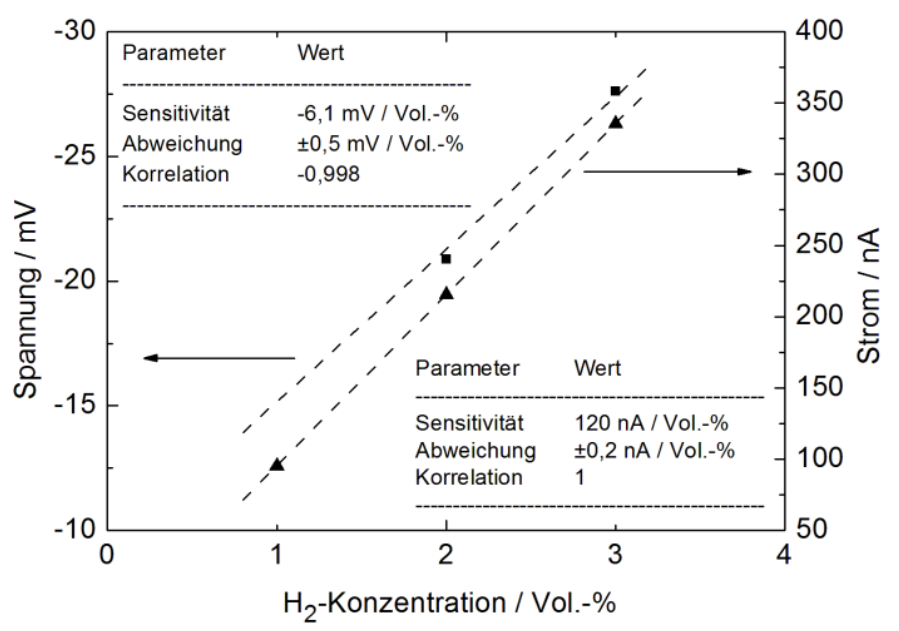

Bild 4 Kalibierkurven des bifunktionalen Gelöstwasserstoffsensors.

\section{Ausblick}

Die erzielten Resultate zeigen die Realisierbarkeit der Kombination zweier Transducerstrukturen zum Nachweis von Gelöstwasserstoff. Zukünftig soll an Stelle der stationären Aufnahme von Kapazitäts/Spannungs-Kurven die Messung in einem dynamischen Modus erfolgen. Somit könnte die lokale pHWertänderung der kapazitiven EIS-Struktur kontinuierlich neben der amperometrischen Messung erfasst werden. Desweiteren soll die Begasung in eine Durchflussanordnung übergeführt werden, um eine direkte Kopplung an einen Laborbiogasreaktor zu ermöglichen.

\section{Danksagung}

Die Autoren danken dem Bundesministerium für Bildung und Forschung (BMBF) für die finanzielle Unterstützung im Rahmen des Förderprojektes „EMSiG“.

\section{Literatur}

[1] H. Neumann, G. Lewitzki, R. Ernst: Multitalent Biogas - Neue Chancen für Landwirtschaft, Industrie und Umwelt. Berlin: Sunbeam GmbH und Solarpraxis AG (2008)

[2] P. Schüsseler: Zielsetzung des Fachgesprächs „Messen-Steuern-Regeln bei der Biogaserzeugung“. In: Gülzower Fachgespräche - Messen, Steuern, Regeln bei der Biogaserzeugung Band 27 (2007), S. 8-16

[3] M. J. Schöning, D. Brinkmann, D. Rolka, C. Demuth, A. Poghossian: CIP (cleaning-in-place) suitable „non-glass“ $\mathrm{pH}$ sensor based on a $\mathrm{Ta}_{2} \mathrm{O}_{5}$-gate EIS structure. Sens. Actuators B 111 (2005), S. 423-429

[4] A. Poghossian: The super-Nernstian pH sensitivity of $\mathrm{Ta}_{2} \mathrm{O}_{5}$-gate ISFETs. Sens. Actuators B 7 (1992), S. 367-370 